\title{
Visualization of the internal flow properties and the material exchange interface in an entraining viscous Newtonian gravity current
}

\author{
Belinda Bates · Christophe Ancey • Jean Busson
}

Received: 3 January 2013/Accepted: 16 June 2013

C) Springer Science+Business Media Dordrecht 2013

\begin{abstract}
In order to simulate a simple entraining geophysical flow, a viscous Newtonian gravity current is released from a reservoir by a dam-break and flows along a rigid horizontal bed until it meets a layer of entrainable material of finite depth, identical to the current. The goal is to examine the entrainment mechanisms by observing the interaction between the incoming flow and the loose bed. The sole parameter varied is the initial volume of the gravity current, thus altering its height and velocity. The gravity current plunges or spills into the entrainable bed and the velocity of the flow front becomes linear with time. The bed material is directly affected: motion is generated in the fluid far downstream of, and in that lying beneath the encroaching front. Shear bands are identified, separating horizontal flow downstream from flow with a strong vertical component close to the step. Downstream of the step the flow is horizontal and stratified, with no slip on the bottom boundary and very low shear near the surface. Between these two regions may lie transitional zones with linear velocity profiles, separated by horizontal bands of high shear; the number of transitional zones in the cross-section varies with the initial volume of the dam-break.
\end{abstract}

Keywords Avalanche dynamics · Debris flows · Entrainment · Viscous gravity currents · Dam-break

\section{Introduction}

Our experimental campaign aims to investigate the mechanisms of entrainment by a viscous gravity current in the simplest case of a Newtonian fluid flowing over an entrainable bed of the same composition. In order to keep the entrainable material in place these experiments had to be conducted at $0^{\circ}$ of inclination. Whereas other studies have investigated entraining gravity currents by observing such a flow from a side-wall $[5,12]$, the present experimental

B. Bates $(\varangle) \cdot$ C. Ancey · J. Busson

Laboratoire d'Hydraulique Environnementale, Ecole Polytechnique Fédérale de Lausanne,

1015 Lausanne, Switzerland

e-mail: belinda.bates@epfl.ch 
configuration visualises a vertical slice of the system in the streamwise direction, at the centre of a channel, thus minimizing any effects that side-walls have been shown to exert on flows atop loose layers [9]. The interface between the entraining current and the initially stationary bed is highlighted and fluid velocity fields are found from particle image velocimetry (PIV), which was possible due to fluorescent tracer particles in the fluid illuminated by a vertical laser sheet.

A review of the existing knowledge about entraining geophysical flows is presented in Sect. 1.1, the experimental set-up and method are described in Sect. 2, and the results and analysis are presented in Sect. 3. Section 3 is split into four parts: first the interface between the gravity current and the initially stationary bed is tracked, then the velocity and shear fields are discussed with reference to entrainment and deposition behaviour, and finally the variation in flow characteristics due to different released volumes is highlighted. Special attention is paid to the regions in the vertical plane where the bed fluid is accelerated from stationary by the released gravity current, and where the incoming fluid is decelerated and therefore deposited due to the presence of the bed fluid.

\subsection{State of current entrainment research}

Geophysical gravity currents such as avalanches and debris flows belong to a special class of event, in which a mixture of solids and fluids (e.g. debris and mud, snow and air) flow as a liquid and may run-out much farther than expected over a slope less steep than the angle of repose. Such phenomena are potentially very dangerous due to their high mobility and have historically led to widespread damage to property and loss of lives [2,25].

Avalanching masses are frequently made more destructive by the process of entrainment. The flow travels over ground covered by loose material, which is incorporated into the flow body, causing the mass to grow substantially. This may or may not alter the rheology of the material, but the bulk dynamics are often significantly affected, with velocities and run-out distances increasing, sometimes many fold [27,32,31].

Exchange of material between the overriding flow and a bed layer underneath is known to affect the characteristics of such a flow, yet is it not well understood how this plays a part due to the difficulties of seeing inside a flowing mass. For example it is disputed whether the addition of stationary mass from below requires a momentum exchange which slows the flowing mass, or whether such a layer of potentially mobile material reduces the friction and thus provokes an increase of velocity.

Different forms of entrainment by snow avalanches have been identified with the aid of radar $[13,32]$. These are: ploughing, where large amounts of material are entrained at the front of the avalanche, bed fluidization by increased pore pressure due to impact, abrasion along the base, and step entrainment where a protective layer of snow is removed, allowing layers of weakly cohesional snow beneath to be entrained almost instantaneously. The present work focuses on basal entrainment and ploughing.

Most models for avalanches and debris flows are derived from the equations for conservation of mass and momentum of a continuum at the macroscopic scale. When entrainment and deposition occur within the system, this must be included in the form of a mass source or sink term [1], and a variation of the basal boundary, as follows [8]:

$$
\frac{d}{d t} \int_{V} \rho d V=Q
$$




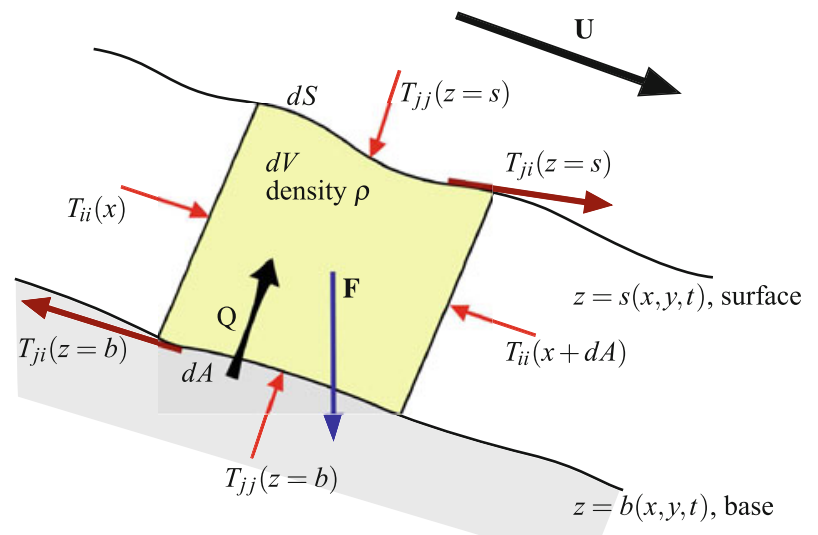

Fig. 1 Origin of the equations of motion for a flowing avalanche based on an infinitesimal column of volume $d V$ and basal surface area $d A$ including a source term $Q$ due to basal entrainment

$$
\begin{aligned}
\frac{d}{d t} \int_{V} \rho \mathbf{u} d V & =\int_{V} \mathbf{F} d V+\int_{S} \mathbf{T} d S, \\
Q=\int_{S} \rho\left(\mathbf{u}_{\mathbf{R}} \cdot \mathbf{n}\right) d S & =\int_{A} E \rho|\mathbf{u}| q d A,
\end{aligned}
$$

with

$$
\mathbf{n}=\frac{1}{q}\left(\frac{\partial b}{\partial x}, \frac{\partial b}{\partial y},-1\right)^{T},
$$

the unit normal to the bed, and

$$
q=\sqrt{\left(\frac{\partial b}{\partial x}\right)^{2}+\left(\frac{\partial b}{\partial y}\right)^{2}+1},
$$

the inclination of the tangent plane to $b$ with respect to the horizontal. Here, as shown in Fig. $1, b(x, y, t)-z=0$ refers to the bed surface elevation, $\mathbf{F}$ to the body forces acting on the volume $d V, \mathbf{T}$ to the sum of all of the stresses acting on the surface $d S, Q$ to the source or sink of mass and $E$ is a yield rate which is positive for entrainment and negative for deposition. The infinitesimal quantity $d A$ represents the surface area beneath the column in contact with the underlying material. $\mathbf{u}_{\mathbf{R}}$ represents the velocity relative to the volume considered. Usually these equations are somewhat simplified by ignoring the cross-flow direction and using the shallow-water assumption, that the length scale is much greater than the height scale, allowing a depth averaging of the horizontal velocity as shown below [14].

$$
\begin{aligned}
\frac{\partial h}{\partial t}+\frac{\partial h \bar{u}}{\partial x} & =E, \\
\frac{\partial h \bar{u}}{\partial t}+\frac{\partial h \bar{u}^{2}}{\partial x} & =g h \sin \theta-g h \cos \theta \frac{\partial h}{\partial x}-\bar{u} E-\frac{\tau_{b}}{\rho}, \\
\frac{\partial b}{\partial t} & =-E .
\end{aligned}
$$


It is still uncertain how to define $E$, and many different forms for this mass exchange parameter have been proposed in the literature, for example Eglit and Demidov [11] discuss a linear dependence on flow velocity $u$, a linear dependence on the flow depth $h$, a dependence on the bed shear stress, proportionally to the flow velocity squared. Cannon and Savage [7] note that more mass will be lost from the bulk at gentler slopes, on rougher terrain and in wider-angled V-channels. Iverson [17] proposes an entrainment parameter proportional to the inverse of the flow velocity, like $1 / \bar{u}$. The volume entrained, according to Chen et al. [8], should vary proportional to the product of the surface area affected and the flow velocity.

More complex models have also been formulated with a mixing layer between the avalanche and the bed, designed to accelerate entrained particles from rest to the velocity of the bulk, e.g. Le and Pitman [19], who described this thin layer using the kinetic theory of particle collisions. Even they concede that "a detailed description of physics at the interface is lacking".

Entrainment effects on avalanching flows have been identified in the lab. A transition from flow over a rigid bed to flow over an erodible bed leads to increased flow resistance caused by a difference in shear stress distribution, thus altering the velocity and the velocity gradient at the bed [10]. Slope angle was seen to be a controlling factor in the effects of entrainable material [22], with a critical angle of $12^{\circ}$ identified, below which entrainment decreases runout and above which it may increase run-out by $40 \%$. Above the critical angle, the thickness of the bed was the limiting parameter. The bed thickness was also identified as important by a numerical study [21].

The study of field data is another approach to the estimation of erosion and entrainment mechanisms. Pierson [27] used data from ten large debris flows triggered by snow-covered volcano eruptions to conclude that debris-flow bulking depended on channel bed erodibility, shear stress exerted on the bed, and the volume of entrainable material. Sovilla et al. [32] noted that entrainment was mainly limited by the entrainable snow, and less by the characteristics of the avalanching snow.

Often, well-documented avalanche and debris-flow events are back-calculated using numerical schemes supplemented by an entrainment law, to see which method produces the results most closely resembling the real events $[20,24,30]$. The drawback of this strategy is that the yield rate or entrained volume in the models is generally selected by the user, based on the events to be simulated, and this parameter is very difficult to estimate [15]. Nevertheless it is recognized that the inclusion of a law describing entrainment and deposition in such models is essential for the better prediction of the dynamics of real avalanches and debris-flows [32].

Recent field campaigns have allowed the in situ study of entrainment and erosion in debris flows, thanks to some innovative technology installed at commonly affected sites. Schurch et al. [28] found a correlation between debris-flow depth and erosion, as the flow depth has an effect on the basal stresses and the hydraulic pressure which are linked to higher erosion rates. They noted also that most of the erosion takes place near the flow front, where the current height is greatest. Berger et al. [6] used a scour sensor to measure erosion rates during debris flows and they found that entrainment of sediment occurred progressively, and was correlated with high pressure and pressure fluctuations near the flow front, caused by particle impacts. McCoy et al. [23] also found progressive entrainment at the flow front, as well as during more dilute flows, but they found no correlation with flow depth and other bulk flow properties. Instead they found high entrainment rates to be caused by high-frequency pore-pressure fluctuations near the surface when the bed sediment was saturated. This agrees with the results of Iverson et al. [18] who found that entrainment of bed sediment was predominantly controlled by sediment saturation as excess pore pressure was generated in the fluid matrix. 


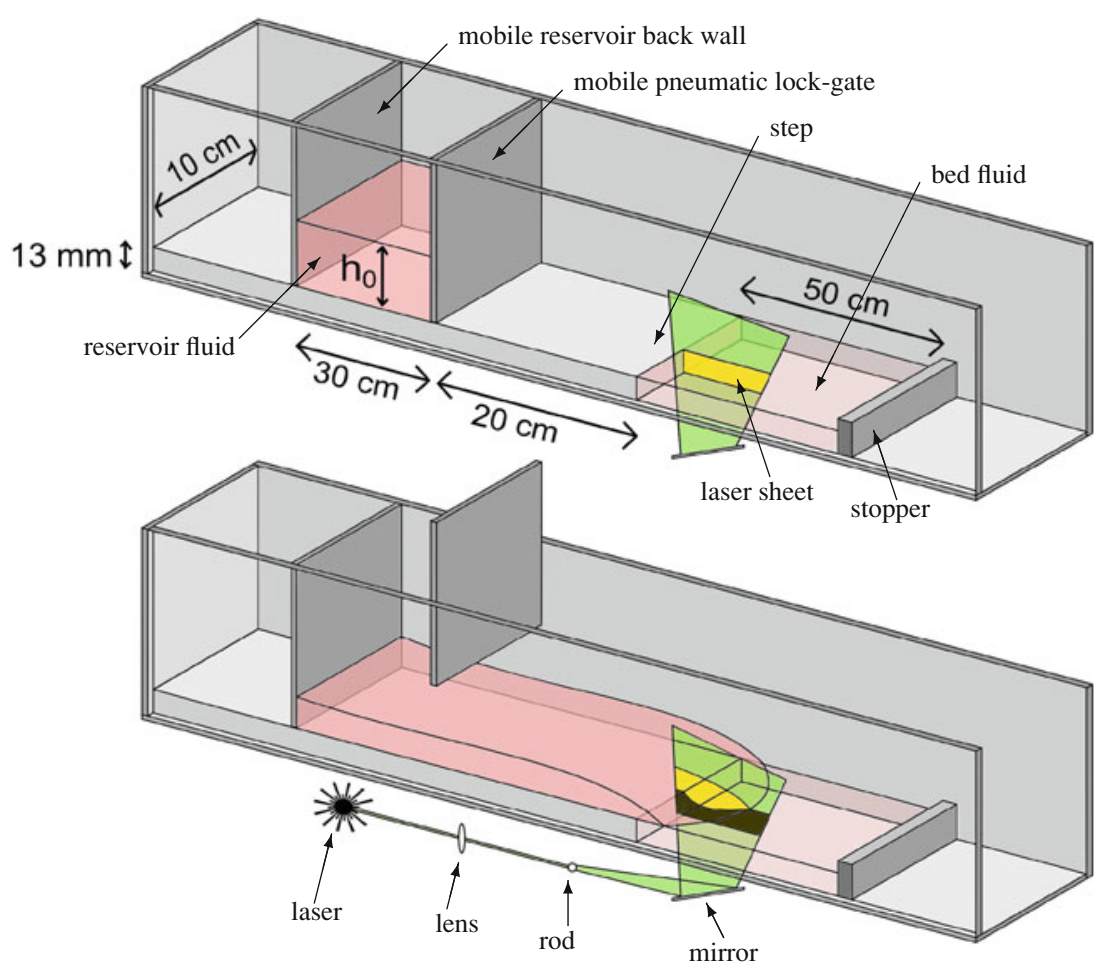

Fig. 2 Schematic showing flume set-up including reservoir, lock-gate, step and bed, and the location of the laser sheet. In the upper image the fluid is contained in the reservoir and the dimensions are shown. The lower image illustrates a flow in motion, detailing the optical set-up (lens, rod and mirror). Here the reservoir fluid is heavily dyed with rhodamine and the bed fluid is lightly dyed, leading to a bright yellow overriding current, and an almost black bed when illuminated by the laser

\section{Experimental configuration and methodology}

\subsection{Flume configuration}

An inclinable flume $3.5 \mathrm{~m}$ long and $10 \mathrm{~cm}$ wide has been modified to include a step and a mobile lock-gate. The details about the original flume can be found in [3,4]. The flume comprises a transparent, roughened PMMA base which allows the use of a laser sheet, projected from below to illuminate a slice of fluid in the vertical-streamwise plane containing a fluorescent dye such as rhodamine. The illuminated plane runs down the centre of the flume, far from the side-walls. The laser is a Diode-Pumped Solid State Nd:YAG Laser with wavelength $532 \mathrm{~nm}$ and maximum power $2 \mathrm{~W}$ and the beam is directed by a lens, rod and mirror beneath the flume (see Fig. 2). The step was added on the flume bottom so that a dambreak flow, released from a reservoir behind a pneumatic lock-gate initially flowed over a rigid bed, made from the same material as the flume, then encountering an entrainable bed of the same height, composed of the same material as was released, as shown in the schematic of Fig. 2. The positions of the reservoir back wall and the lock-gate were adjustable so that the reservoir could be moved closer to or further away from the entrainable bed and observation window. This allowed flows to develop sufficiently before they reached the step, yet minimizing travel time. 
Table 1 Fluid and image acquisition properties for each run

\begin{tabular}{|c|c|c|c|c|c|c|}
\hline & \multicolumn{6}{|c|}{ Volume released } \\
\hline & $300 \mathrm{ml}$ & $400 \mathrm{ml}$ & $500 \mathrm{ml}$ & $600 \mathrm{ml}$ & $700 \mathrm{ml}$ & $800 \mathrm{ml}$ \\
\hline \multicolumn{7}{|l|}{ Dye configuration } \\
\hline Reservoir only & $300 \mathrm{R}$ & $400 \mathrm{R}$ & $500 \mathrm{R}$ & $600 \mathrm{R}$ & $700 \mathrm{R}$ & $800 \mathrm{R}$ \\
\hline Bed only & $300 \mathrm{~B}$ & 400B & $500 \mathrm{~B}$ & $600 \mathrm{~B}$ & $700 \mathrm{~B}$ & $800 \mathrm{~B}$ \\
\hline Combined & $300 \mathrm{C}$ & $400 \mathrm{C}$ & $500 \mathrm{C}$ & $600 \mathrm{C}$ & $700 \mathrm{C}$ & $800 \mathrm{C}$ \\
\hline \multicolumn{7}{|l|}{ Camera properties } \\
\hline Exposure time (ms) & 7.6517 & 6 & 6 & 5 & 5 & 4.5 \\
\hline Frame period (ms) & 60 & 35 & 20 & 10 & 9.6 & 9.6 \\
\hline Acquisition time (s) & 56 & 30 & 15 & 10 & 10 & 10 \\
\hline \multicolumn{7}{|l|}{ Fluid properties } \\
\hline Solution concentration & \multicolumn{6}{|c|}{$98.5 \%$ glycerol by weight; $1.5 \%$ distilled water } \\
\hline Fluid viscosity (Pas) & \multicolumn{6}{|l|}{1.0445} \\
\hline Fluid density $\left(\mathrm{g} \mathrm{cm}^{-3}\right)$ & \multicolumn{6}{|l|}{1.2572} \\
\hline Fluid temperature $\left({ }^{\circ} \mathrm{C}\right)$ & \multicolumn{6}{|l|}{20} \\
\hline
\end{tabular}

\subsection{Fluid properties}

The fluid released from the reservoir was a glycerol-water solution at $98.5 \%$ concentration by weight. The flume properties were set as follows: the step height and therefore entrainable bed depth was $13 \mathrm{~mm}$. Six hundred fifty milliliters of fluid was placed downstream of the step, making up a loose bed of $50 \mathrm{~cm}$ in length, with a stopper at the far end. This fluid is referred to as bed fluid. The length of the adjustable reservoir was kept constant at $30 \mathrm{~cm}$, with the pneumatic gate placed $20 \mathrm{~cm}$ away from the step, where the current entered the entrainment zone. The fluid released from the reservoir was of variable volume and therefore depth, ranging from 300 to $800 \mathrm{ml}$, thus creating dam-break flows of variable height and velocity at the step. This was designed to test how the speed and height of the gravity current at entry into the loose bed would affect the way the material was entrained. Table 1 details the different experiments conducted, the camera set-up, and the fluid material properties: density, viscosity and temperature.

For each volume of fluid released, we used three configurations to visualise the behaviour. One involved a high concentration of dye in the reservoir fluid and very little in the bed fluid, this is referred to as a "reservoir run" and denoted by the letter R as in Fig. 3 (top). The idea here was to have detailed information about the flow characteristics within the current that enters the bed, whilst showing a clear interface between the initially stationary bed fluid and that released from the reservoir. The opposite set-up was named a "bed run" denoted by a B, which gives detail about the bed fluid, whilst still showing the interface (Fig. 3, bottom left). Finally a "combined run", denoted by a C (Fig. 3, bottom right), gave details about all fluid in the system at the same time, but the interface was not shown thus the information about the origins of each fluid was lost. Figure 3 also highlights the difference in shape of the interface for different released volumes, and shows the refraction problems we experienced when larger volumes of fluid were released, due to the increased curvature of the flow descending the step. 

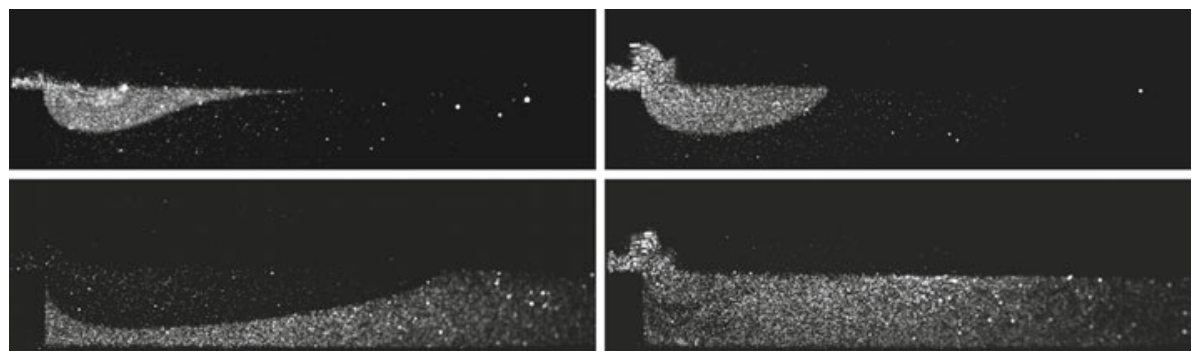

Fig. 3 Raw images (contrast adjusted for illustration): above left $300 \mathrm{ml}$ dyed fluid released into undyed bed (300R). Above right $600 \mathrm{ml}$ dyed fluid released into undyed bed (600R). Below left $500 \mathrm{ml}$ undyed fluid released into dyed bed (500B). Below right $500 \mathrm{ml}$ dyed fluid released into dyed bed (500C)

After conducting experiments with all three configurations described above, it was clear from the analysis of the results that the most useful runs were the $\mathrm{R}$ runs in which dyed fluid entered a predominantly undyed bed. This is not to say that the other experiments were not useful, as they provided verification of velocity measurements in darker regions of the images and qualitative descriptions of both the entire flow and the flow of the bed material alone. However the R runs allowed PIV analysis of the entire system-due to the small amounts of dye present in the bed fluid-along with the tracking of the interface between the reservoir and bed fluid.

\subsection{Image acquisition and analysis}

A high-speed camera (Basler A403k with Nikkon Micro Nikkor $53 \mathrm{~mm}$ lens and orange filter) was situated underneath the flume and took consecutive images of the flow as it made the transition from a standard gravity current to flow over an entrainable bed. Photos were obtained through a transparent prism, employing the Scheimpflug principle which allowed visualisation of the vertical laser sheet without any problems associated with the free surface curvature of a gravity current. The size of the observation window was $80 \mathrm{~mm}$, but the light from the laser only illuminated a region around $60 \mathrm{~mm}$ long accurately enough for reliable measurements.

After consideration of various techniques, velocities were calculated using PIV between two images, five frames apart (better for removing spurious vectors), and then, with the exception of the first two velocity profiles, averaged over five frames (so for image $i$, the mean velocities from $i-2, i-1, i, i+1$ and $i+2$ were used). The velocity field for the first image of each run was not averaged, and for the second image it was averaged over the first three frames. Shear profiles were calculated using PIV between two consecutive images, as too much data was lost when using larger jumps. All measurements were corrected for perspective using a calibration grid. The PIV package used was an opensource software called MatPIV [33].

\section{Results and discussion: visualization of the flow properties}

The PIV method described above gives an array of vertical and horizontal velocities at grid coordinates, based on correlations of pixel patterns in interrogation windows between two images. From this it was possible to describe the velocity and shear fields in the fluid. The interface between bed and reservoir fluid was identified from the differential colouring of the respective fluids - in the R runs the reservoir fluid glowed much more brightly due to its higher 
concentration of rhodamine tracers, and a sequence of MatLab image operations allowed the isolation of this interface (composed of two lines: the deepest extent of the interface base, and the furthest extent of the interface front). The interface location was subject to small errors (of the order of a few pixels), yet sometimes when a large dyed particle from the bed was picked up further downstream, this gave an inaccurate reading and was subsequently ignored. This happened only rarely, so on the whole we can be confident about the interface location measurements.

\subsection{Interface tracking}

Using image operations to identify the interface we were able to describe the motion of the gravity current as it entered the entrainable bed in the central vertical-streamwise plane. One of the runs $(300 \mathrm{ml})$ was very slow reaching the observation window and a contact line instability had developed before bed entry. This meant that the fluid front first entered the bed off-centre, and thus the bed fluid was already in motion before we saw the front begin entrainment in the observation plane. The other runs with larger released volumes did not suffer from this problem.

For all runs, the reservoir fluid displaced the bed fluid in a progressive way, with the deepest displacement near the step, and a shallow region nearest the horizontal flow front (hereafter referred to as the nose). This nose was pointed for smaller released volumes (300, $400 \mathrm{ml}$ ) and blunt for larger volumes (>500 ml) as shown in Fig. 3 (top), but this may have been due to the instability described above where the front in the central plane spilled out onto a bed which already had a streamwise velocity. The larger currents plunged into the entrainable bed, with large initial downwards velocity. This difference between spilling and plunging flows should be treated with caution, but it may signal a regime change between lesser and greater effects of vertical motion of the current.

The deepest position of bed fluid displaced by the reservoir fluid at any time $t$ is described as $d_{\max }(t)$, and is interpreted as the erosion depth for the viscous avalanche case, the same concept as the $d_{e}$ defined by Gauer and Issler [13] for two phase snow-air avalanches. Figure 4 (top) shows the dimensionless values $D_{\max }$ plotted against dimensionless $t^{*}=t / t_{c}$, where $t_{c}$ is the recorded time taken for the front to travel to the edge of the step. It was chosen to scale time with the measured travel time, instead of with a length to buoyancy velocity ratio because the currents took longer than expected to reach the step, and we can perhaps attribute this to the intermediate state the system is in (traditional time scales being defined for long and short times). The depth scaling identified which allowed the graphs to collapse on a similar curve is as follows:

$$
D_{\text {scale }}=\frac{v}{\sqrt{g^{\prime} d}}\left(\frac{l_{T}}{l_{0}}\right)^{2 / 3},
$$

where $v=\mu / \rho$ is the dynamic viscosity in $\mathrm{m}^{2} \mathrm{~s}^{-1}, g^{\prime}$ is the reduced gravity in $\mathrm{m} \mathrm{s}^{-2}$, taking into account the density of the ambient air, $d$ is the step height $0.013 \mathrm{~m}, l_{T}$ is the total step length $0.5 \mathrm{~m}$ and $l_{0}$ is the initial reservoir length $0.3 \mathrm{~m}$. Time was non-dimensionalised by $t_{c}$, as described above. This depends of course on the initial volume of fluid released, whereas the depth scaling is $\approx 3.272 \mathrm{~mm}$, independent of the volume released. Curves were fitted to this data, showing that at early times the maximum erosion depth increased like $\left(t^{*}\right)^{1 / 2}$ and at later times like $\left(t^{*}\right)^{1 / 5}$. Examining the velocity profiles around the time of this change in power law shows that the behaviour of the entraining current near the step changes from highly erosional to less erosional. In other words the fluid near the interface between the step and the location of $d_{\max }$ slows down (including in some cases the fluid inside the interface) 

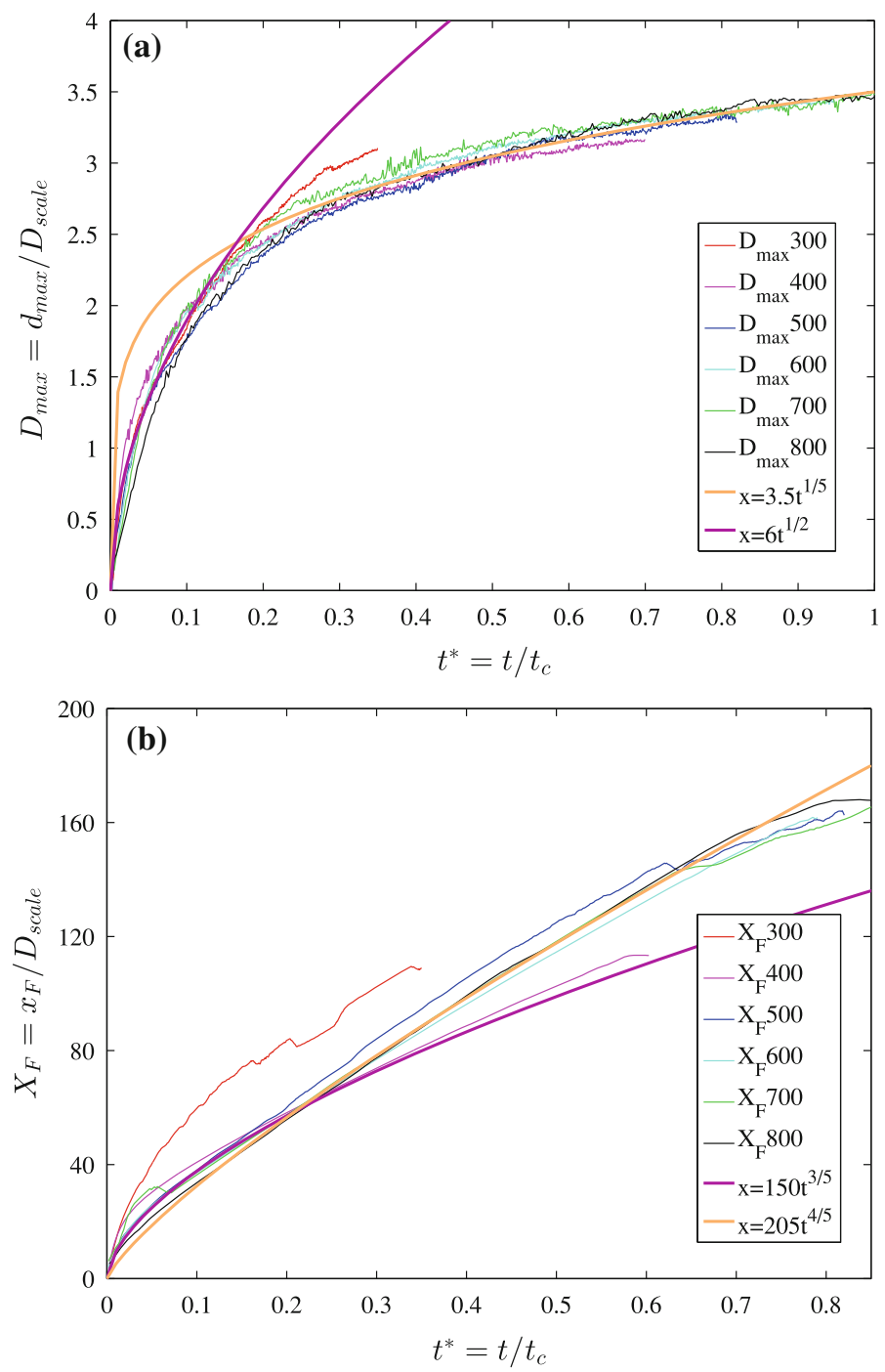

Fig. 4 a Progression of dimensionless maximum depth of displaced region with dimensionless time $D_{\max }\left(t^{*}\right)$, for all released volumes, 300-800 ml. $D_{\text {scale }}=\left(v / \sqrt{g^{\prime} d}\right)\left(l_{T} / l_{0}\right)^{2 / 3}$. Fitting curves show that $D_{\max }$ scales with $\left(t^{*}\right)^{1 / 2}$ at early times and $\left(t^{*}\right)^{1 / 5}$ at later times. b Progression of the dimensionless front position of the gravity current in the bed with dimensionless time $X_{F}\left(t^{*}\right)$, for all released volumes, 300-800 $\mathrm{ml}$. Fitting curves show that $X_{F}$ scales with $\left(t^{*}\right)^{3 / 5}$ at early times and $\left(t^{*}\right)^{4 / 5}$ at later times

showing the start of deposition in this region. We had thought that $d_{\max }$ would be limited by the finite depth of the entrainable material in the case of the larger currents, however the non-dimensionalised $D_{\max }$ shows a similar progression in all cases.

In the same way the front of the interface, or the furthest downstream point, $x_{F}(t)$ was identified. This was non-dimensionalised with the same length scale as above $D_{\text {scale }}$, and tracked with time, as shown in Fig. 4 (bottom). The data collapse is less conclusive than for the maximum erosion depth due to measurement uncertainties, but the data can be fitted fairly well by a $\left(t^{*}\right)^{3 / 5}$ rule at early times, and by a $\left(t^{*}\right)^{4 / 5}$ rule at later times. The two exceptions to 

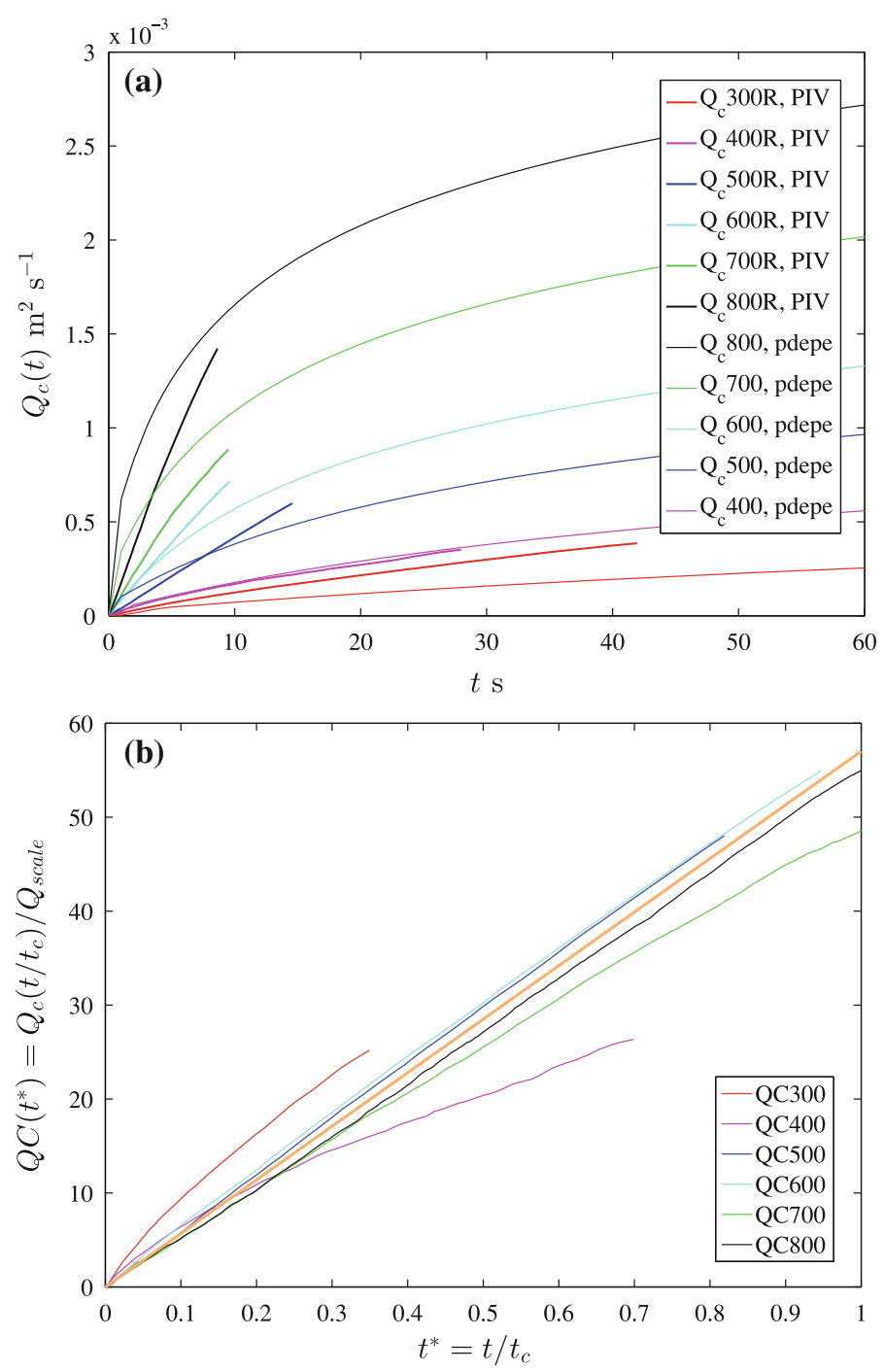

Fig. 5 a Measured cumulative discharge per unit width, $Q_{c}(t) \mathrm{m}^{2} \mathrm{~s}^{-1}$ (thick lines), compared with the theoretical values (fine lines) computed at the step by MatLab solver pdepe, based on the solver by Skeel and Berzins [29]. b Dimensionless cumulative discharge per unit width with dimensionless time $Q C\left(t^{*}\right)=$ $Q_{c}\left(t / t_{c}\right) / Q_{\text {scale }} \cdot Q_{\text {scale }}=1,000\left(D_{\text {scale }}\right)^{2}\left(h_{0} / l_{0}\right)^{10 / 3}$. Fitted with straight line

this rule are $X_{F} 300$ and $X_{F} 400$, those produced by the 300 and $400 \mathrm{ml}$ dam-breaks. This is a possible consequence of the fact that these fronts exhibited a slightly different entrainment behaviour, spilling rather than plunging into the bed.

Using PIV on a small region of the flow above the step allowed the calculation of the volume influx $Q(t)=\bar{u}\left(x_{\text {step }}, t\right) h\left(x_{\text {step }}, t\right)$ by identification of the height and the depth averaged velocity of the gravity current close to the end of the step. Comparing the measured cumulative discharge per unit width, $Q_{c}(t) \mathrm{m}^{2} \mathrm{~s}^{-1}$, with the theoretical values computed at the same location on a rigid bed using Huppert's formula [16] shows that the presence of a step and an entrainable bed completely alters the discharge behaviour. When plotted with 
time, almost all of the runs produced a linear rule with time for the measured $Q_{c}(t)$ (thick lines), yet the theoretical solutions (thin lines) are curves following a series of power laws (Fig. 5, top). The dimensionless values $Q C\left(t^{*}\right)$ were found by non-dimensionalising with the scaling $1,000\left(D_{\text {scale }}\right)^{2}\left(\frac{h_{0}}{l_{0}}\right)^{10 / 3}$ and the data collapse approximately onto a straight line as shown in Fig. 5 (bottom). The two flows that depart slightly from this trend are the smallest released currents (the 300 and $400 \mathrm{ml}$ cases) with the smallest velocities. Due to the slowness of the currents, a small amount of fluid was lost down the side of the step during run-up to the entrainable bed, and we have also seen that the entraining front behaved differently in these cases, spilling rather than plunging. These are two possible explanations for the slight discrepancy.

\subsection{Velocity profiles}

The picture is more complicated than just tracking the interface moving inside the entrainable bed. The entry of the gravity current into the loose bed stimulated large-scale mobility of the fluid at rest, and so it was useful to map the entire velocity profile, using arrows to show direction and magnitude and colour contours for the absolute velocity $U(x, y, t)=$ $\sqrt{u(x, y, t)^{2}+v(x, y, t)^{2}}$, where $u(x, y, t)$ is the fluid velocity in the horizontal direction and $v(x, y, t)$ is in the vertical direction. The interface is included in the images for illustrative purposes.

Figure 6 displays a sequence of velocity profiles within our vertical cross-section starting from the first entrance of the gravity current into the bed. The case chosen for illustration is $700 \mathrm{R}$, where $700 \mathrm{ml}$ of dyed reservoir fluid was released into an undyed bed. The fluid enters from the left hand side, above the step, shown as a white rectangle. The encroaching fluid front released from the reservoir (interface shown here in black) set the bed fluid in motion, below and far downstream of the front, as it was displaced by the front's plunging motion. Images are taken $9.6 \mathrm{~ms}$ apart, so if image 1 represents time $t=0 \mathrm{~s}$, image 10 was $0.096 \mathrm{~s}$ later, image $200.192 \mathrm{~s}$ later, image $400.384 \mathrm{~s}$ later, image $1201.152 \mathrm{~s}$ later, image $2802.688 \mathrm{~s}$ later and image $4704.512 \mathrm{~s}$ later. Streamlines are also shown in Fig. 7 for images and image 120 .

We see that even in image 10 the bed fluid was mobilised in a region much larger than the incoming front, shown by cyan to red regions outside the interface. This shows entrainment by a ploughing mechanism. Early on, vertical velocities were not negligible, as shown by the streamlines of image 40 in Fig. 7. Later on, some of the fluid from the reservoir slowed right down, represented by dark blue regions inside the interface, close to the step. This shows deposition in our system.

Between these two end cases, the flow far from the step became horizontally stratified, with little to no vertical velocity: flow near the surface was fast and almost uniform, and fluid close to the base was slow to stationary. This horizontal flow is clearly shown by the streamlines of image 120 in Fig. 7. In the region close to the step there was a high velocity zone near the surface, shown by red regions in Fig. 6. Just downstream of this zone lay the most strongly vertical velocity vectors, yet further downstream the flow had no vertical velocity component. The following subsection shows that these flow regions were separated by regions of strong shear.

The lack of slip on the step wall and the base led to the development of a stagnant zone in the corner. In fact there were slowly recirculating eddies here, like cavity flow in a corner [26] but our techniques only showed this at very long times. 
$U \mathrm{~mm} \mathrm{~s}^{-1}$

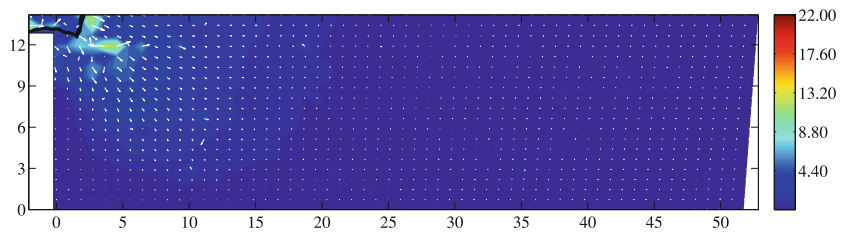

$t=0 \mathrm{~s}$

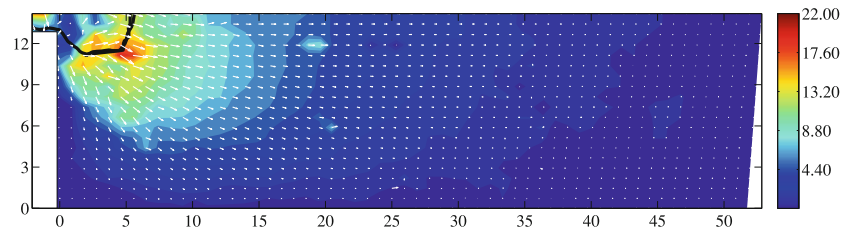

$t=0.096 \mathrm{~s}$

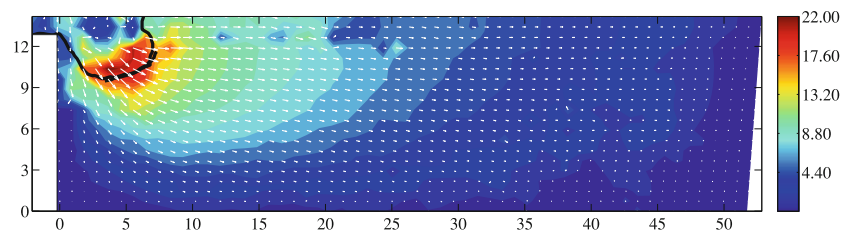

$t=0.192 \mathrm{~s}$

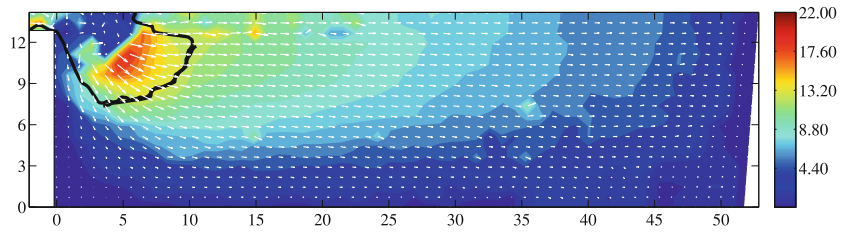

$t=0.384 \mathrm{~s}$

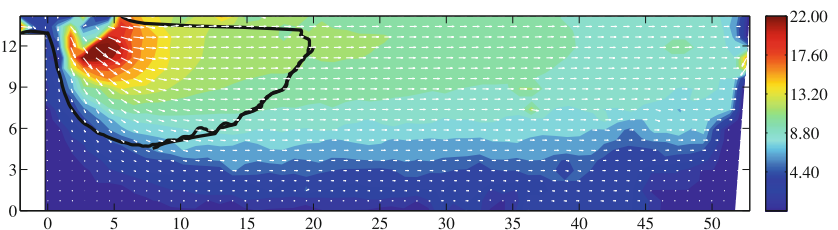

$t=1.152 \mathrm{~s}$

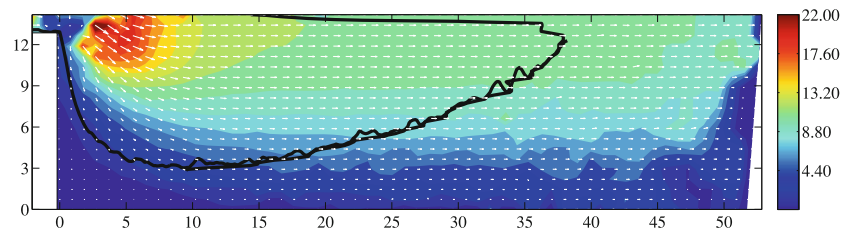

$t=2.688 \mathrm{~s}$

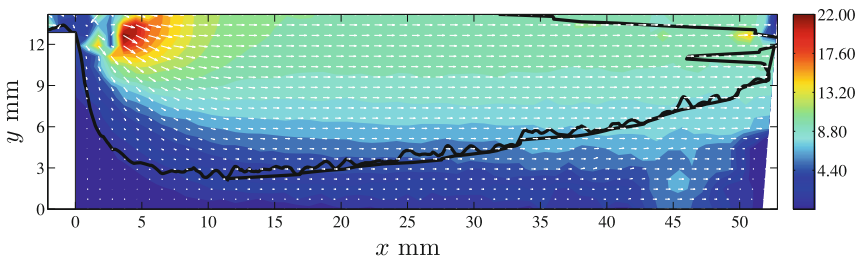

Fig. 6 Development of absolute velocity field, calculated by PIV, for run 700R. Measurements in $\mathrm{mm} \mathrm{s}^{-1}$. Interface between bed fluid and incoming reservoir fluid shown in black. $13 \mathrm{~mm}$-high step shown in white 


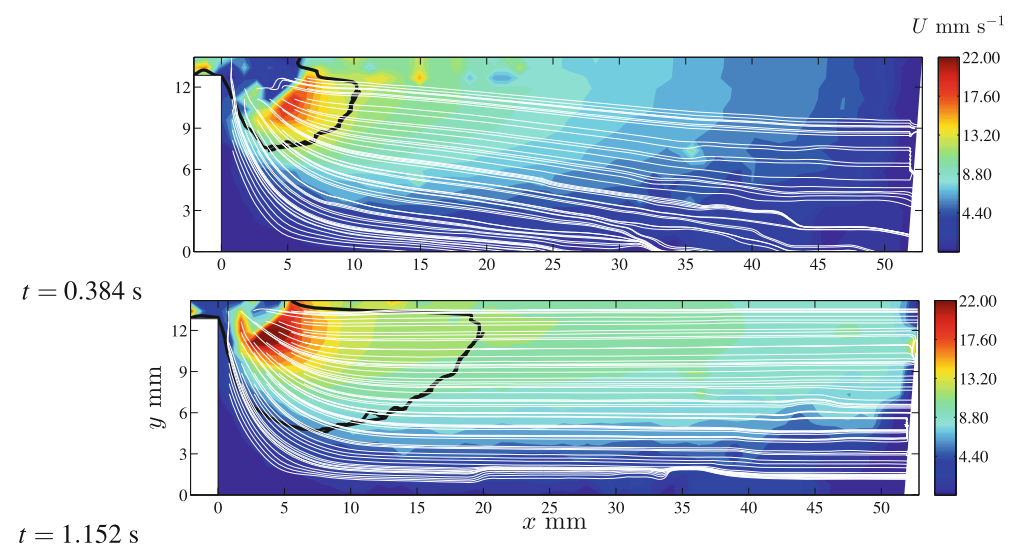

Fig. 7 Some approximate streamlines calculated from the PIV data superimposed onto the velocity field

\subsection{Shear profiles}

Due to the large quantities and the complex nature of the velocity data obtained from the experiments, it was useful to simplify the analysis by searching for flow zones in the streamwise flow section. The structure of the velocity field was examined more closely by considering the second invariant of the rate-of-strain tensor:

$$
I_{2}(x, y, t)=\sqrt{\left|2 \frac{\partial u}{\partial x} \frac{\partial v}{\partial y}-\frac{1}{2}\left(\frac{\partial u}{\partial y}+\frac{\partial v}{\partial x}\right)^{2}\right|},
$$

often seen to represent the magnitude of shear.

Figure 8 shows $I_{2}(x, y, t)$ in $\mathrm{s}^{-1}$ on our vertical streamwise section for the same 700R run. The most striking thing about these images is that upon entry of the gravity current into the entrainable bed, two shear bands appeared, which grew in the downstream direction, but did not migrate vertically (except after the fluid had entered the depositional regime, when the upper band migrates upwards). The gravity current then propagated into the region already defined by these dynamical characteristics.

The interpretation of these shear bands is that they separate flow zones in the verticaldownstream plane. Near the surface and far downstream there was minimal shear, defining the uniform flow zone which has already been identified in the velocity profiles of Fig. 6 . Close to the step and near the rigid base there was a corner of fluid with little to no shear. This is the region in which the corner eddies developed on the long time scale, but on the time scale of the flow this was a stationary region. The slow flow zone in this corner extended along the rigid base where viscous fluid obeyed the no-slip condition.

Between the surface flow region and the slow flow region is where the two shear bands were located. Fluid in motion moved on top of the fluid stuck to the rigid base, creating the lower shear band. Between this and the upper shear band was a transitional region where the velocity profile was roughly linear (see Fig. 9 for an example), and the upper shear band separated this zone from the uniform flow above. This middle layer can be interpreted as a transition zone, where the fluid was accelerated by the fluid in motion above it due to horizontal viscous shear stresses acting along the base of the upper layer. Fluid next to the 


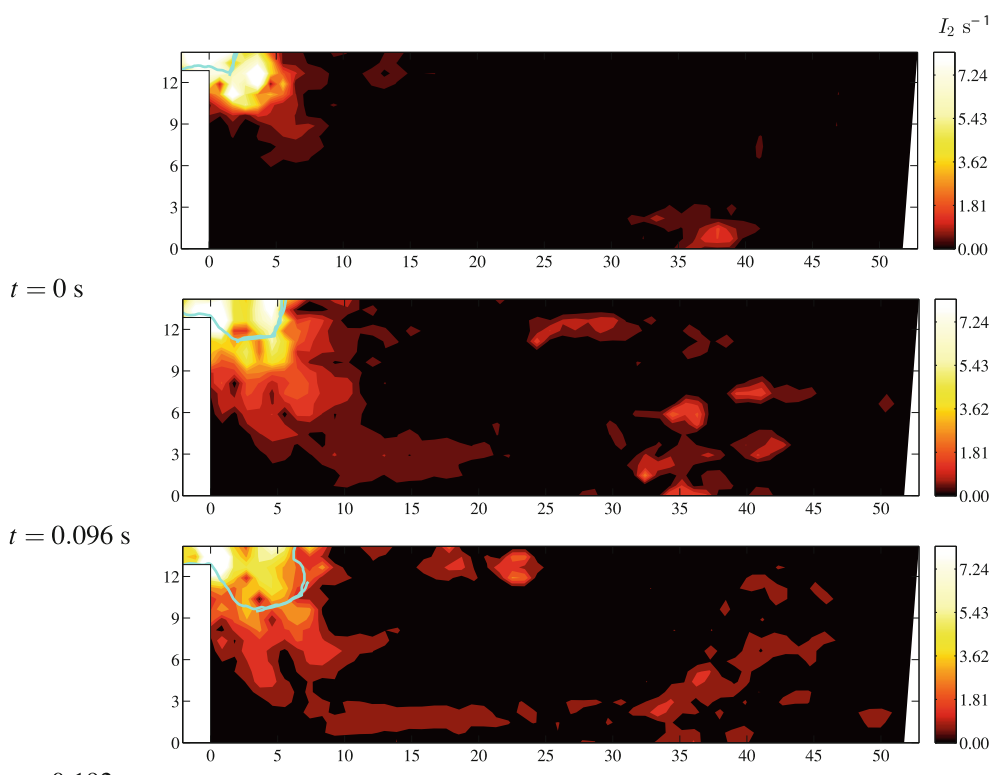

$t=0.192 \mathrm{~s}$

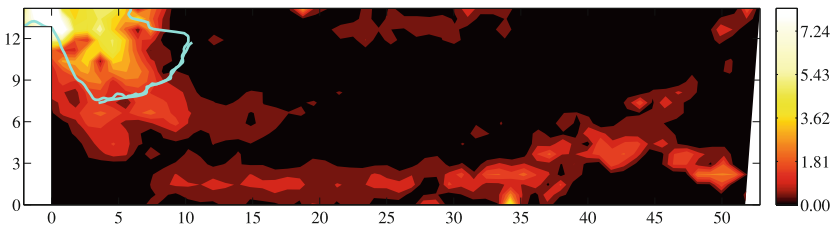

$t=0.384 \mathrm{~s}$

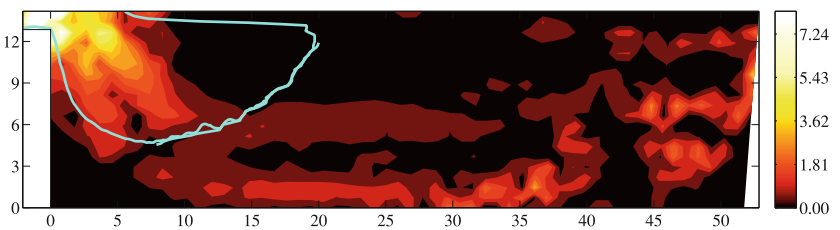

$t=1.152 \mathrm{~s}$

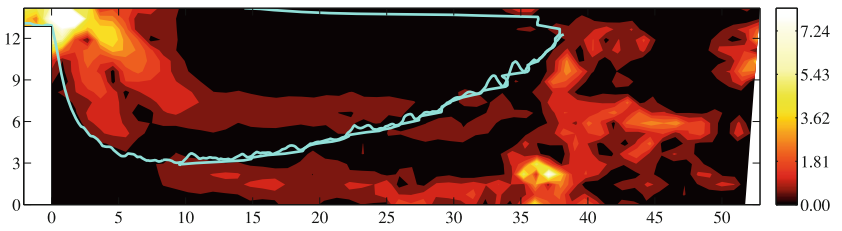

$t=2.688 \mathrm{~s}$

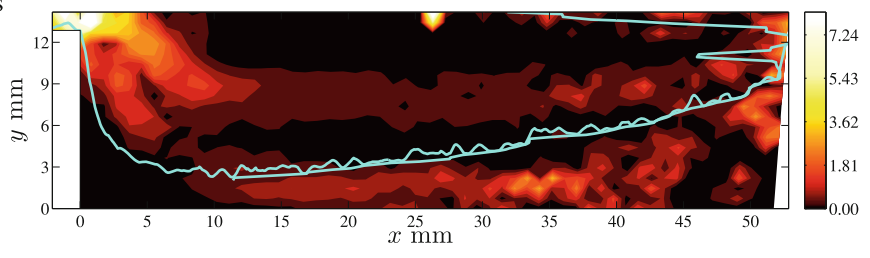

$t=4.512 \mathrm{~s}$

Fig. 8 Development of the second invariant of the rate-of-strain tensor for run 700R. Measurements in $\mathrm{s}^{-1}$. Interface between bed fluid and incoming reservoir fluid shown in cyan. 13 mm-high step shown in white 


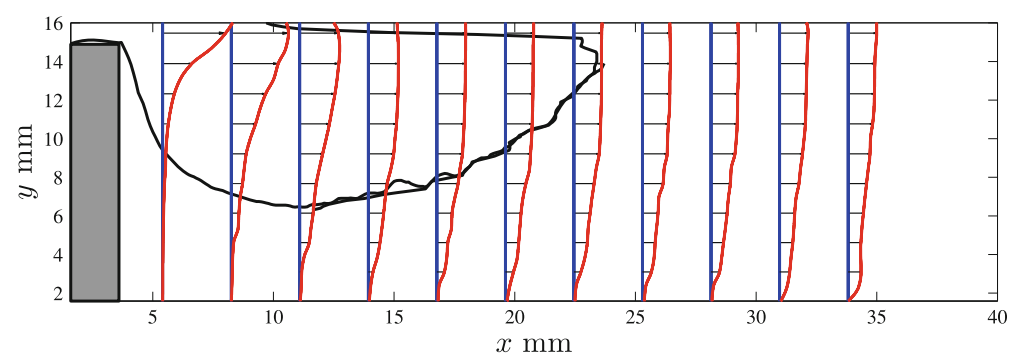

Fig. 9 Variation of horizontal velocity with height and distance downstream for run 700R, image 120, 1.152 s after initial entry of current into bed. Step shown on left in grey, interface between reservoir and bed fluid shown by black line

base was kept in place by the lack of slip. Thus the stationary fluid was entrained stepwise into the flow by a mechanism similar to that of abrasion in real granular avalanches.

Bearing in mind that the gravity current studied was a simple viscous Newtonian fluid, this transition layer is reminiscent of the mixing layer in Le and Pitman [19]; and the stepwise entrainment of debris in field studies [6,23] allows a qualitative comparison with our findings.

Another shear zone was found between the surface flow close to the step and further downstream. This shear arose from the fact that velocities near the surface were predominantly vertical close to the step, yet just the other side of the high velocity region, shown as red in Fig. 6, the fluid velocity was strongly horizontal. Fluid with a strong vertical velocity tended to be deposited, and if it recirculated it did so in one of the transitional zones, not in the uniform surface flow.

\subsection{Variation of flow structure with released volume}

The depth of the faster moving layer, displaced by the ploughing motion of the incoming fluid, varied with volume released. Larger gravity currents mobilised a deeper layer of fluid, extending almost to the rigid base, and smaller currents had little effect on the bottom fluid. Thus recirculating eddies were only visible in the flows of 700 and $800 \mathrm{ml}$, however they may have just been too slow to identify in the smaller experiments.

The number of shear bands in the vertical cross-section varied with released volume, as illustrated in Fig. 10. For intermediate released volumes, 600 and $700 \mathrm{ml}$, an upper and a lower shear band were present, with one transition zone between the slow flow at the base and the uniform upper flow. For the smallest released volumes, 300-500 ml, only one shear band was identified separating nearly stationary fluid from the almost uniform flow above. For the largest volume, $800 \mathrm{ml}$, three shear bands were present, showing two transition zones. All of this indicates that the nature of such stepwise entrainment, caused by the overriding fluid in the upper layer and the no-slip condition at the base, depends on the released volume. The number of steps necessary to accelerate the material from rest was dependent on the incoming flow velocity, which in this case was a direct consequence of the volume of the fluid in the reservoir before the dam-break.

A summary of the differences in flow properties caused by the variation of released volume is given in Table 2. Here $R e_{1}$ refers to the time-averaged Reynolds number of the flow before the step, and $R e_{2}$ is the time-averaged Reynolds number of the flow after the step. These numbers are estimates using the flow height as a length scale. The value $t_{c}$ represents the time taken by the current to reach the step, $20 \mathrm{~cm}$ from the lock-gate, which was used for the 

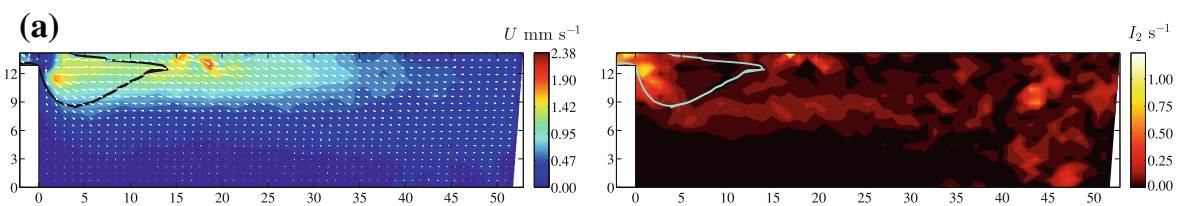

(b)
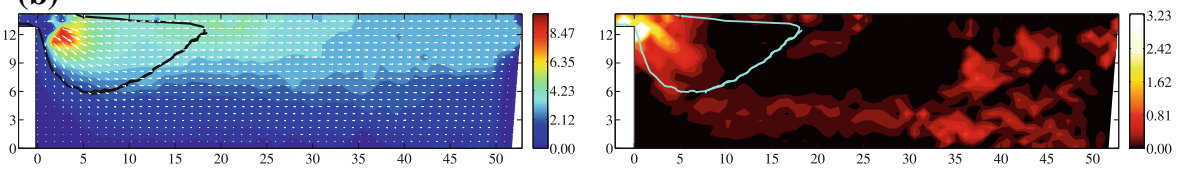

(c)
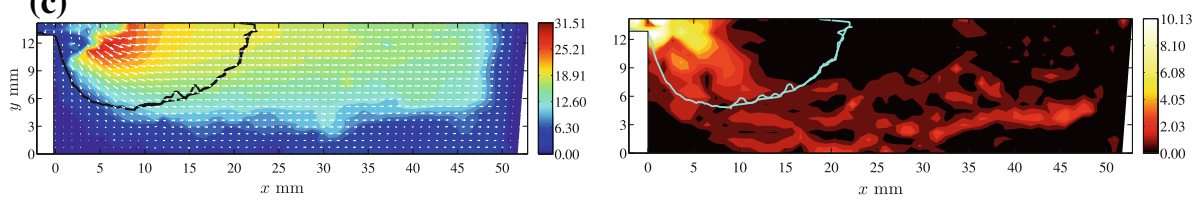

Fig. 10 Other events for comparison. a $300 \mathrm{ml}$ released, image 100 taken $6 \mathrm{~s}$ after fluid entry: one shear band; b $500 \mathrm{ml}$ released, image 150 taken $3 \mathrm{~s}$ after fluid entry: one shear band; c $800 \mathrm{ml}$ released, image 90 taken 0.864 s after fluid entry: three shear bands

Table 2 Flow properties for each released volume: $R e$ before and after step, time taken to reach the step, typical horizontal velocity of the interface nose, scaling for $Q_{c}(t)$, number of shear bands

\begin{tabular}{lccccccc}
\hline $\begin{array}{l}\text { Volume } \\
\text { released (ml) }\end{array}$ & Initial & $R e_{1}$ & $R e_{2}$ & $t_{c}(\mathrm{~s})$ & $u_{F}\left(\mathrm{~mm} \mathrm{~s}^{-1}\right)$ & $Q_{\text {scale }}\left(\mathrm{m}^{2}\right)$ & $\begin{array}{l}\text { Number of } \\
\text { shear bands }\end{array}$ \\
\hline 300 & 10.0 & 0.01 & 0.01 & 120.00 & 0.96 & 0.128 & 1 \\
400 & 13.3 & 0.02 & 0.01 & 40.00 & 1.32 & 0.333 & 1 \\
500 & 16.6 & 0.05 & 0.04 & 17.78 & 3.95 & 0.701 & 1 \\
600 & 20.0 & 0.09 & 0.07 & 10.12 & 6.49 & 1.286 & 2 \\
700 & 23.3 & 0.11 & 0.09 & 5.65 & 12.14 & 2.150 & 2 \\
800 & 26.6 & 0.19 & 0.09 & 3.35 & 19.21 & 3.356 & 3 \\
\hline
\end{tabular}

non-dimensionalisation of time, the values $u_{F}$ are typical values for the horizontal velocity of the nose of the interface between reservoir and bed fluid. The sixth column is the scaling used to non-dimensionalise $Q_{c}(t)$, the cumulative discharge at the step, and the final column shows the number of shear bands in the vertical profile for the flow.

\section{Conclusion}

\subsection{Sequence of events}

The initial entrance of a viscous Newtonian gravity current into an entrainable bed quickly mobilised a deep layer of material. This is equivalent to a ploughing mechanism as the encroaching front plunged into the bed, entraining material in the surface layer far downstream. The material mobilised in this way developed into a region of uniform horizontal flow, with the flow front (the nose of the overriding current) moving at an almost constant speed 
in all runs. The depth of this region was dependent on the volume released, however it was probably limited by the depth of the bed in the experiments where the larger gravity currents were released. It was bounded below by a shear layer in which the velocities strongly varied with perpendicular distance from the rigid wall (far from the step the horizontal shear rate was important, close to the step both the vertical and horizontal velocities were significantly sheared).

\subsection{Transition zones in the vertical plane}

Depending on the volume of fluid released from the reservoir, a number of regions lay between this shear band and the rigid bed. For the smallest released volumes, the upper layer sheared on top of a slow layer which extended all the way down to the base with no further distinct regions. For larger released volumes, transition zones, where the velocity profile was linear, lay between two or more shear bands. These transition zones facilitated the faster motion of the uniform surface flow layer, while fluid near the rigid base still satisfied the no-slip condition. Increasing the dam-break volume and thus the velocity of the gravity current increased the number of transition zones generated in the entrainable bed.

A high shear region was also found at the beginning of the entrainable zone, separating the horizontal flow region far from the step from a more strongly downwards moving region close to the step. This fluid moving close to the step was eventually deposited.

\subsection{Displacement of bed material}

The maximum depth of fluid displaced by the front, or the non-dimensional erosion depth, $D_{\max }$ follows a $t^{1 / 2}$ rule at early times, and $t^{1 / 5}$ thereafter. This change in power law seems to correspond with the entrainment activity of the current becoming weaker close to the step, and in some cases with the start of some depositional behaviour. The maximum horizontal front position $x_{F}$ displaces bed material at a rate proportional to $t^{3 / 5}$ at early times, and $t^{4 / 5}$ thereafter. The cumulative discharge of reservoir material follows a linear rule with time, contrary to that predicted using Huppert's model for a gravity current, thus the presence of the entrainable bed significantly changes the flow characteristics.

It is noted that the depth of the loose material may have had an effect, as the height of the step was kept constant. A numerical study should be conducted to examine the effects of a deeper bed, and to see if the same number of shear bands and transition zones are reproduced. As the applications of this work are to debris flows and avalanches, where the entrainable material is in a finite and often shallow layer, further experiments will be conducted to examine the effects of a shallower bed of varying length.

Acknowledgements This work was supported by funding from Ecole Polytechnique Fédérale de Lausanne. Many thanks to Nicolas Andreini, Gaël Epely-Chauvin and Bob de Graffenried for their help and guidance during this project.

\section{References}

1. Adduce C, Sciortino G, Proietti S (2012) Gravity currents produced by lock-exchanges: experiments and simulations with a two layer shallow-water model with entrainment. J Hydraul Eng 138(2):111-121

2. Ancey C, Gervasoni C, Meunier M (2004) Computing extreme avalanches. Cold Reg Sci Technol 39:161180

3. Andreini N (2012) Dam break of Newtonian fluids and granular suspensions. PhD Thesis, EPFL, Switzerland 
4. Andreini N, Epely-Chauvin G, Ancey C (2012) Internal dynamics of Newtonian and viscoplastic fluid avalanches down a sloping bed. Phys Fluids 24(5):053101

5. Barbolini M, Biancardi A, Cappabianca F, Natale L, Pagliardi M (2005) Laboratory study of erosion processes in snow avalanches. Cold Reg Sci Technol 43(1-2):1-9

6. Berger C, McArdell BW, Schlunegger F (2011) Direct measurement of channel erosion by debris flows, Illgraben, Switzerland. J Geophys Res 116:F01002

7. Cannon SH, Savage WZ (1988) A mass-change model for the estimation of debris-flow runout. J Geol 96(2):221-227

8. Chen H, Crosta GB, Lee CF (2006) Erosional effects on runout of fast landslides, debris flows and avalanches: a numerical investigation. Geotechniques 56(5):305-322

9. Delannay R, Louge M, Richard P, Taberlet N, Valance A (2007) Towards a theoretical picture of dense granular flows down inclines. Nat Mater 6(2):99-108

10. Egashira S, Itoh T, Takeuchi H (2001) Transition mechanism of debris flows over rigid bed to over erodible bed. Phys Chem Earth B 26(2):169-174

11. Eglit ME, Demidov KS (2005) Mathematical modeling of snow entrainment in avalanche motion. Cold Reg Sci Technol 43(1-2):10-23

12. Estep J, Dufek J (2012) Substrate effects from force chain dynamics in dense granular flows. J Geophys Res 117:F01028

13. Gauer P, Issler D (2004) Possible erosion mechanisms in snow avalanches. Ann Glaciol 38:384-392

14. Gray JMNT (2001) Granular flow in partially filled slowly rotating drums. J Fluid Mech 441:1-29

15. Hungr D, Evans SG (2004) Entrainment of debris in rock avalanches: an analysis of a long run-out mechanism. Geol Soc Am Bull 116(9-10):1240-1252

16. Huppert HE (1982) The propagation of two-dimensional and axisymmetric viscous gravity currents over a rigid horizontal surface. J Fluid Mech 121:43-58

17. Iverson RM (2012) Elementary theory of bed-sediment entrainment by debris flows and avalanches. J Geophys Res 117:F03006

18. Iverson RM, Reid ME, Logan M, LaHusen RG, Godt JW, Griswold JP (2011) Positive feedback and momentum growth during debris-flow entrainment of wet bed sediment. Nat Geosci 4(2):116-121

19. Le L, Pitman EB (2009) A model for granular flows over an erodible surface. SIAM J Appl Math 70(5):1407-1427

20. Luna BQ, Remaitre A, van Asch TWJ, Malet JP, van Westen CJ (2012) Analysis of debris flow behavior with a one dimensional run-out model incorporating entrainment. Eng Geol 128:63-75

21. Mangeney A, Tsimring LS, Volfson D, Aranson IS, Bouchut F (2007) Avalanche mobility induced by the presence of an erodible bed and associated entrainment. Geophys Res Lett 34:L22401

22. Mangeney A, Roche O, Hungr O, Mangold N, Faccanoni G, Lucas A (2010) Erosion and mobility in granular collapse over sloping beds. J Geophys Res 115:F03040

23. McCoy SW, Kean JW, Coe JA, Tucker GE, Staley DM, Wasklewicz TA (2012) Sediment entrainment by debris flows: in situ measurements from the headwaters of a steep catchment. J Geophys Res 117:F03016

24. McDougall S, Hungr O (2005) Dynamic modelling of entrainment in rapid landslides. Can Geotech J 42(5):1437-1448

25. Melosh HJ (1986) The physics of very large landslides. Acta Mech 64:89-99

26. Moffatt HK (1964) Viscous and resistive eddies near a sharp corner. J Fluid Mech 18:1-18

27. Pierson TC (1995) Flow characteristics of large eruption-triggered debris flows at snow-clad volcanosconstraints for debris-flow models. J Volcanol Geotherm Res 66(1-4):283-294

28. Schurch P, Densmore AL, Rosser NJ, McArdell BW (2011) Dynamic controls on erosion and deposition on debris-flow fans. Geology 39(9):827-830

29. Skeel RD, Berzins M (1990) A method for the spatial discretization of parabolic equations in one space variable. SIAM J Sci Stat Comput 11:132

30. Sovilla B, Bartelt P (2002) Observations and modelling of snow avalanche entrainment. Nat Hazards Earth Syst Sci 2:169-179

31. Sovilla B, Sommavilla F, Tomaselli A (2001) Measurements of mass balance in dense snow avalanche events. Ann Glaciol 32:230-236

32. Sovilla B, Burlando P, Bartelt P (2006) Field experiments and numerical modeling of mass entrainment in snow avalanches. J Geophys Res 111:F03007

33. Sveen JK (2004) An introduction to matpiv v. 1.6.1. Technical report, University of Oslo 